\title{
LOCALISED HYPERTROPHY OF THE SEMIMEMBRANOSUS MUSCLE SIMULATING POPLITEAL CYST
}

\author{
Pan. P. Symeonides and Constantine Paschaloglou, Athens, Greece \\ From the Department of Orthopaedics, Royal Hellenic Air Force Hospital, Athens
}

A cyst is the comomnest cause of swelling in the popliteal fossa (Baker 1885; Cravener 1932; Burleson, Bickel and Dahlin 1956). Other causes include lipoma, enlarged lymph nodes, aneurysm of the popliteal artery and neuromas. The purpose of this paper is to describe a circumscribed hypertrophy of the semimembranosus muscle presenting as a swelling in the popliteal fossa and simulating a popliteal cyst (Wilson, Eyre-Brook and Francis 1938; Haggart 1943). We have had the opportunity to observe four such cases, in two of which operation was undertaken. So far as we are aware this cause of popliteal swelling has not previously been reported.

\section{CASE REPORTS}

Case 1-An airman aged twenty complained of a painless swelling in the inner half of the popliteal fossa. It had appeared about three years before his first attendance and gradually increased in size. It caused no symptoms except for a sensation of tension in the popliteal region on walking.

Examination revealed a soft, non-tender, fairly well circumscribed swelling 6 centimetres long and 4 centimetres wide: its height depended on the degree of flexion of the knee, reaching a maximum of 3 to 4 centimetres. The range of knee movement was normal. Radiographs did not show any abnormality of the bones. A similar swelling of about equal size was palpated in the left popliteal fossa.

Operation and progress-At operation the right popliteal space was explored under general anaesthesia. Despite meticulous search of the structures of the area no abnormality was found. The patient was discharged after an uneventful post-operative course. No definite diagnosis was made. The swelling was present practically unchanged when we saw the patient one and three months after operation.

Case 2-An airman aged nineteen was referred to the out-patient clinic for swellings behind both knees of four years' duration (Figs. 1 and 2). Although they were slowly enlarging they caused no symptoms.

On examination, egg-sized swellings were present in the medial half of both popliteal fossae. There was no tenderness on palpation and no sign of inflammation. Knee movements were not restricted. During active flexion of the knee the swelling became harder and increased in size.

Operation and progress-Because of our experience in Case 1, we started the operation on the right side under local anaesthesia. A myocele was excluded as the fascia was intact. When the fascia was incised a large fusiform swelling of the semimembranosus muscle appeared on active flexion of the knee (Fig. 3). The muscular part of the semimembranosus extended more distally than usual. The abnormality could then be recognised, even when the muscle was relaxed. The hypertrophic part of the muscle extending longitudinally was removed and the resultant gap was closed by interrupted sutures.

Six months later operation was carried out on the left side. The findings were similar to those described above. Histological examination of both specimens showed only "hypertrophy of muscle fibres".

When the patient was re-examined three and nine months after the second operation no abnormality was found.

VOL. 52 B, NO. 2, MAY 1970 


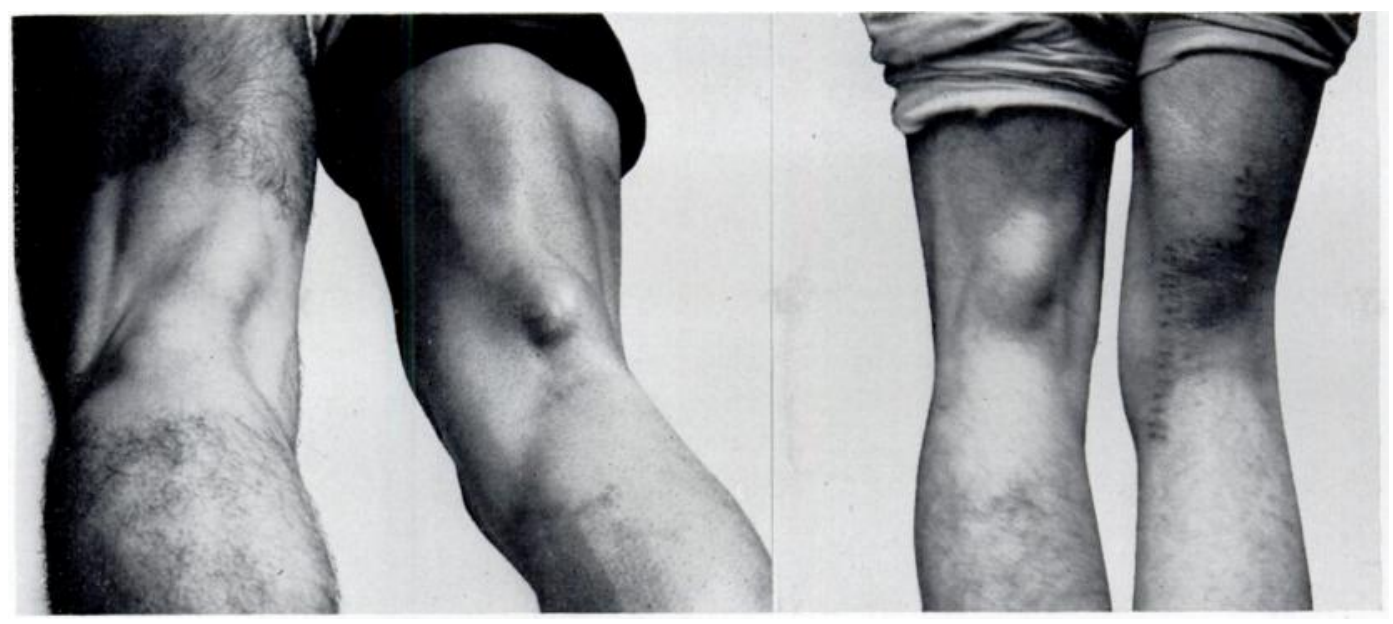

Fig. 1

FIG. 2

Case 2. Figure 1-Before operation. The swelling behind the right knee, which is actively flexed, simulates a popliteal cyst. On the left side the swelling is hardly seen because the knee is not flexed. Figure 2-The swelling is now clearly seen on the left side. Operation had been carried out on the right and the contour of the limb is restored to normal.

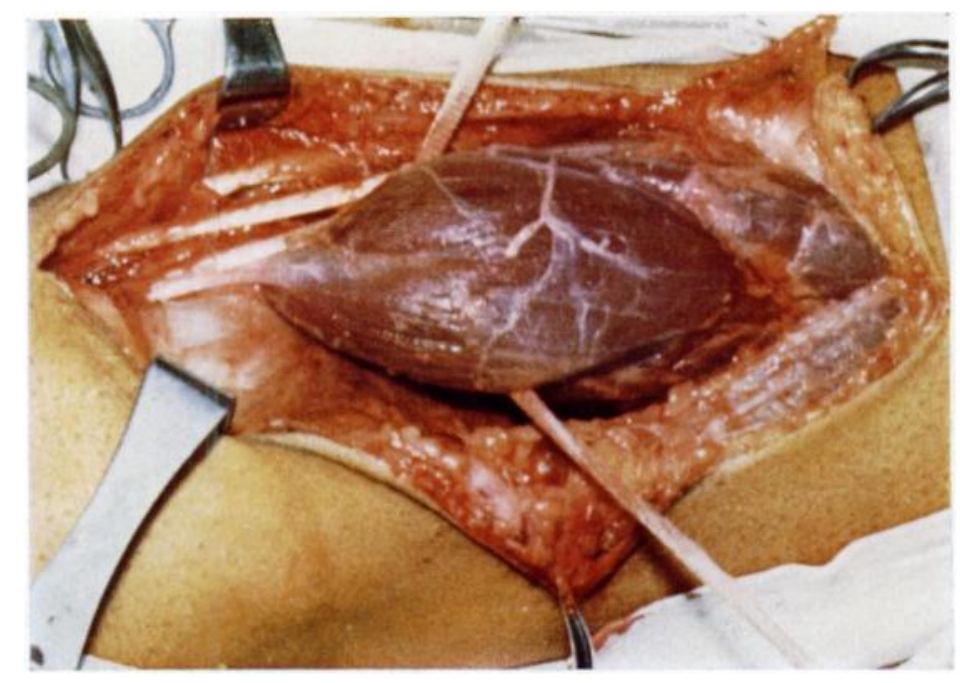

Fig. 3

Case 2 -The large fusiform swelling of the semimembranosus displayed on the right side after incision of the popliteal fascia.

\section{DISCUSSION}

It is clear that the swellings in the popliteal fossae of the patients described were due to simple enlargement of the semimembranosus muscle. The increase in size and hardening of the swelling during active contraction of semimembranosus is consistent with this explanation. This would also explain our failure to identify the lesion in Case 1 during exploration under general anaesthesia.

In the last eight months we have seen two further patients with clinical findings similar to those reported above. Although a definite diagnosis was not made, because operation was not undertaken, we think that they represent additional cases of localised hypertrophy of the semimembranosus muscle for these reasons: they appeared over a period of three to four years in young active subjects; they were painless, lacked other signs of inflammation 
and increased in size during active flexion of the knee. (A popliteal cyst increases in size when the knee is extended.)

Thus it seems that this condition is not rare and should be considered in the differential diagnosis of swellings in the popliteal fossa.

Nothing definite can be said about etiology. The peculiarity of the semimembranosus muscle should be considered in this respect. The muscle is unipennate (Gray 1967) - that is, its fibres are arranged obliquely in relation to the "line of pull" and do not run the whole length of muscle. In such a case the force of the muscle's action is resolved into two components: one acting in the "line of pull" and the other at right angles to it. Thus a great part of its energy is lost, which forces the muscle to hypertrophy locally. The reason why the enlargement appears mainly in the distal part of the muscle could be that the muscular fibres are more oblique in that region. The appearance of the lesion in active young adults is consistent with this hypothesis.

\section{SUMMARY}

1. Two cases of swelling in the popliteal fossa due to a local hypertrophy of the semimembranosus muscle are described and two further cases thought to be of similar nature are noted.

2. The swelling was soft, not tender, fairly well circumscribed, and without signs of inflammation. Its most characteristic feature was an increase in size and hardening of the swelling during active flexion of the knee.

3. This condition should be considered in differential diagnosis of swellings of the popliteal fossa.

\section{REFERENCES}

BAKfr, W. M. (1885): The Formation of Abnormal Synovial Cysts in Connection with the Joints. Saint Bartholomew's Hospital Reports, 21, 177.

Burleson, R. J., Bickel, W. H., and Dahlin, D. C. (1956): Popliteal Cyst. Journal of Bone and Joint Surgery, 38-A, 1265.

Cravener, E. K. (1932): Hernia of the Knee Joint (Baker's Cyst). Journal of Bone and Joint Surgery, 14, 186. GraY, H. (1967): Gray's Anatomy. Thirty-fourth edition, p. 586. Edited by D. V. Davies. London: Longmans. Haggart, G. E. (1943): Synovial Cysts of the Popliteal Space: Clinical Significance and Treatment. Annals of Surgery, 118, 438.

Wilson, P. D., Eyre-Brook, A. L., and Francis, J. D. (1938): A Clinical and Anatomical Study of the Semimembranosus Bursa in Relation to Popliteal Cyst. Journal of Bone and Joint Surgery, 20, 963. 